\title{
Is sexology necessary in dermatology? Review of most common sexual dysfunction in skin disorders
}

\author{
Mariusz Jaworski \\ Department of Medical Psychology, Medical University of Warsaw, Warsaw, Poland
}

Corresponding author: Mariusz Jaworski, PhD, E-mail: mjaworski@wum.edu.pl

\begin{abstract}
Dermatology not only treats diseases, but also is the study of the skin, especially its structures. Many studies which showed a lot of patients' psychological problems in dermatological diseases were reported, but there is still too small number of papers about sexual problems of these patients. The aim of this study was the analysis of sexual problems in patients with dermatological diseases. To be included in the review, a study had to: (i) Human data; (ii) concerned patients' sexual problems in skin diseases; (iii) concerned the patient-doctor communication in the context of human sexuality; (iv) be published in an English-language journal. Studies were identified through online database searches of PUBMED, MEDLINE, Web of Knowledge (2000-2016). As a result of review, there was observed dominant number of publications about patients' sexual problems in psoriasis, and significantly less in other skin diseases such as acne, atopic dermatitis, chronic hand eczema. The most common sexual problems in skin disease were: sexual dysfunction, especially erectile dysfunction; some problems in relationship and sexual behaviour. It should be noted that all reported publication about sexual problems in skin disorders were focused on adult patients. There is still too small publication in adolescents.
\end{abstract}

Key words: Sexual; Sexology; Psoriasis; Acne; Atopic dermatitis

\section{INTRODUCTION}

Dermatology is a part of medicine which examines and treats skin diseases. Among the most common skin disorder are: acne, atopic eczema, benign tumours and vascular lesions, contact dermatitis and other eczemas, leg ulcers, skin cancer, psoriasis, and viral warts. It should be stress that dermatology not only treats diseases, but also is the study of the skin, especially its structures [1].

It should be stressed that there were many studies which showed a lot of patients' psychological problems in dermatological diseases. In this case, anxiety, depression, and other psychological problems, which affect patients' lives and psychosocial well-being, were reported [2].

In the latest literature attention is drawn to the role of skin changes during dermatological diseases in patients' interpersonal relations. This is because many skin diseases are associated with severity of skin changes which are very often visible. Moreover, skin is important in human aesthetics and appearance. Because of this, the skin plays key roles for interpersonal communication, and real-life collaboration, because it is involved in emotional expression. Skin conductivity is sensitive to many different stimuli such as strong emotion, a startling event, pain, exercise, deep breathing, a demanding task, etc. [2,3].

It should be stress that non-verbal communication gives a lot of information, because all messages can be communicated and understood through hand and arm gestures, body language or posture, eye contact (or its lack), touch, facial expression, as well as dress code, hairstyles or smells. There are some specialists who stress that non-verbal communication is the most powerful form of communication, and it giving an opportunity to go in other persons mind. According to this, it is very

\footnotetext{
How to cite this article: Jaworski M. Is sexology necessary in dermatology? Review of most common sexual dysfunction in skin disorders. Our Dermatol Online. 2017;8(3):341-347.

Submission: 27.09.2016; Acceptance: 02.12.2016

DOI: 10.7241 /ourd.20173.97
} 
important to know how to use it correctly. There are many forms and tips for learning and improving personal non-verbal skills. Correct interpretation as well as usage of learned skills could be helpful in context of achieving goals on work or medical therapy $[4,5]$.

In the context of non-verbal communication, chronic dermatological conditions have a significant effect on patients' psychological condition, self-esteem and body image. They also affect patients' functioning in all other spheres of life (social, family, professional etc.) and contribute fundamentally to quality of life. Moreover, it could have a negative influence not only on human real-life collaboration, and relationship with another people, but also may unwittingly lead to psychosexual problems in context of human sexuality $[6,7]$.

In sexology, human sexuality is defined as a complex and multidimensional aspect of human life which is associated with biological, psychological, social, and cultural factors. There are some studies which noted that many of human personality traits, as well as behaviour patterns are rooted in the stages of psychosexual development. According to this, It can lead to development of normal or abnormal behaviour which are depending upon the experiences of the individual during psychosexual developmental stages [8].

Dermatological diseases can also have a negative impact on psychosexual development, especially in adolescents and young adults. Is should be stress that both psychosexual development, and personality development are connected. According to the World Health Organization (WHO), sexuality is a basic human need, as well as an important aspect of human beings. This need cannot be separated from others. WHO highlights that sexuality is extremely important in maintaining good mental health $[8,9]$.

\section{Aims}

According to this, the aim of this study was the analysis of sexual problems in patients with dermatological diseases. According to the main aim of this paper, the following research question were formulated:

1. Are patients' sexual problems reported in skin diseases? If yes, What kind of sexual patients' problems are reported most frequently?

2. Are there any patients' dermatological problems in context of human psychosexual development, especially among adolescents?
3. Are there studies which analysed the relationship between the skin changes and development of sexual dysfunction in patients with dermatological diseases?

\section{Search Procedure and Criteria for Study Inclusion}

To be included in the review, a study had to: (i) Human data; (ii) concerned patients' sexual problems in skin diseases; (iii) concerned the patient-doctor communication in the context of human sexuality; (iv) be published in an English-language journal. All types of epidemiological studies regarding sexual function in patients with skin diseases were included. Reviews and case reports were excluded. Full texts of all studies meeting the inclusion criteria were reviewed, and their bibliographic references were checked for additional sources. The variables assessed were as follows: the type of study, sample size, instruments used, statistical analysis and results.

Studies were identified through online database searches of PUBMED, MEDLINE, Web of Knowledge (2000-2016), using the key words: sexual problems, skin disease, sexual dysfunction, dermatological diseases, erectile dysfunction, human sexuality, sexual behaviour, psoriasis, acne, atopic dermatitis, chronic hand eczema, patient-doctor relationship, doctor's communication, medical interview. These key words were combined with each other.

\section{RESULTS}

As a result of review, there was observed dominant number of publications about patients' sexual problems in psoriasis, and significantly less in other skin diseases such as acne, atopic dermatitis, chronic hand eczema. These results were presented in the next part of this publication. It should be noted that all reported publication about sexual problems in skin disorders were focused on adult patients. There is still too small publication in adolescents.

\section{Psoriasis}

Psoriasis is a chronic inflammatory disease, resulting from immune and proliferative changes that affect the skin. The world incidence of this disease is estimated to be $1 \%-3 \%$ [10]. It should be stress that development of psoriasis is associated with a lot of psychosocial factor, such as chronic stress, and several psychological disorders, for instance: depression and anxiety $[11,12]$. 
We identified some epidemiological studies regarding psoriasis and sexual dysfunction. Some researchers stress that there is a strong relationship between psoriasis and human sexual functioning, because this disease causes intense interpersonal strain, and hindering quality of life [13].

It should be noted that sexual problems in psoriasis could be associated with medicamentous therapy or disease development. In the terms of medicamentous therapy, some studies about sexual dysfunction in psoriasis have been reported, especially sexual impotence and erectile dysfunction due to the medicamentous therapy, such as etretinate and methotrexate $[14,15]$. However, there is a study which showed that the risk of sexual dysfunction was not significantly elevated in patients receiving systemic treatment, including retinoid, methotrexate, and cyclosporine [16].

It should be noted that relationship between medicamentous therapy and sexual dysfunction is a very important problem in the medical practice. It is associated with the fact that some prescription drugs can affect patients' sexual function. If patient observes this relationship, he or she could stop taking this medicaments. This situation can have negative influence on all therapy. Many scientists highlight that it has been difficult to address the issue of sexual functioning and prescription drug use, because of many approaches to it.

\section{Sexual dysfunction}

According to the ICD-10 classification, the sexual dysfunction is defined as: "the various ways in which an individual is able to participate in a sexual relationship as he or she wishes'. This classification includes such sexual dysfunction as: Sexual dysfunction, not caused by organic disorder or disease (F52), Lack or loss of sexual desire (F52.0), Sexual aversion and lack of sexual enjoyment (F52.1), Failure of genital response (F52.2), Orgasmic dysfunction (F52.3), Premature ejaculation (F52.4), Nonorganic vaginismus (F52.5), Nonorganic dyspareunia (F52.6), Excessive sexual drive (F52.7), Other sexual dysfunction, not caused by organic disorder or disease (F52.8), and Unspecified sexual dysfunction, not caused by organic disorder or disease (F52.9) [17].

Molina-Leyva et al. [18] carried out a prospective case series study with 80 patients diagnosed with moderate to severe psoriasis, and 80 healthy controls. In this study, authors analysed not only sexual dysfunction, but also problems of anxiety and/or depression, as well as psoriasis' distribution patterns. In the context of sexual dysfunctions, all participants completed the Massachusetts General Hospital-Sexual Functioning Questionnaire (MGH-SFQ). The MGHSFQ questionnaire is consist of 5 items addressing the different phases of the sexual cycle: (1) sexual interest; (2) excitation; (3) orgasm; (4) erection (only in males), and (5) global sexual satisfaction. Each item is scored from 0 (completely reduced) to 4 (normal). High scores mean better sexual functioning. In this study, it was shown that sexual impairment in psoriasis patients occurs in all components of the sexual response such as: sexual interest; excitation; orgasm; and erection (only in males case), as well as global sexual satisfaction. Molina-Leyva et al. [18] observed that the prevalence of sexual dysfunction was $53.7 \%$ in patients with psoriasis. This prevalence was significant higher than in controls (healthy volunteers; $17.5 \%$ ). It should be noted that male patients with psoriasis were characterised by an increase in erectile dysfunction compared to controls. This result suggests that sexual dysfunction in psoriasis are very important problem in medical practice, and it must be a consequence of several combined factors.

Another prospective case series study, which was carried out on 133 patients (44 women; mean age $42.0 \pm 14.1$ and 79 men; mean age $47 \pm 11.7$ ), showed similar results. In this study, authors observed that an increase in sexual dysfunction was associated with psoriasis lesions on abdomen, genitals, lumbar region, and buttocks in context of women, and chest, genitals, and buttocks in context of men [19].

Very interesting observation about sexual dysfunction in psoriasis was made by Chen et al. [20]. It was cohorts study of the 73,800 sampled patients, 12,300 male patients with newly diagnosed psoriasis and 61,500 matching controls from National Health Insurance Database in Taiwan. In this study, authors stressed that the hazard ratio (HR) for sexual dysfunction for psoriasis patients was 1.27 times ( $95 \%$ confidence interval [CI], 1.11-1.46; P =0.001). Moreover, the risk of sexual dysfunction was higher in patients with psoriatic arthritis (HR: 1.78, 95\% CI: 1.08-2.91). This cohorts study has shown that male patients with psoriasis are at increased risk of developing sexual dysfunction. For this reason, doctors should pay more attention to the impact of psoriasis on patients' sexual health.

In cross-sectional study, which was carried out by Maaty et al. [21] in 52 sexually active female psoriasis 
patients and 30 controls, analysed sexual function in female patients with psoriasis. Authors showed a negative correlation between the PASI score and the sexual satisfaction in psoriasis patients. Moreover, the negative correlation between sexual dysfunction and psoriasis was also reported in other studies. For instance, in cross-sectional study of Sampogna et al. [22] in 936 patients with psoriasis; cross-sectional study of Al-Mazeedi et al. [23] in 330 patients with psoriasis; cross-sectional study of Al-Meeuwis et al. [24] in 487 patients with psoriasis; and randomized clinical trial study of Guenther et al. [25] in 1996 patients with psoriasis. In this patients' group, impaired sexual function was reported by $22.6 \%$ (women $=27.1 \%$; men $=20.8 \%$ ). It should be stressed that this sexual dysfunction was significantly associated with increased psoriasis severity. At week 12, the proportion of ustekinumab-treated patients with impaired sexual function decreased from $22.4 \%$ to $2.7 \%$ compared with no change with placebo $(\mathrm{p}<0.001)$.

\section{Erectile dysfunction}

In sexology, erectile dysfunction is defined as: 'the persistent inability to attain and maintain an erection sufficient to permit satisfactory sexual performance'. Moreover, epidemiological data have shown a high prevalence and incidence of ED worldwide. There are many studies which showed that erectile dysfunction may affect human physical and psychosocial health, as well as have a significant impact on the quality of life and their interpersonal relationship. Erection is a complex phenomenon. Because of this, the pathophysiology of erectile dysfunction may be vasculogenic (e.g., Cardiovascular disease and Hyperlipidaemia), neurogenic (e.g., Degenerative disorders and Central nervous system tumours), anatomical (e.g., Peyronie's disease and Hypospadias), hormonal (e.g., Hypogonadism, Hyperprolactinemia, Hyper- and hypothyroidism, Hyper- and hypocortisolism), drug-induced (e.g., Antihypertensives (thiazide diuretics, etc.); Antidepressants (selective serotonin reuptake inhibitors, tricyclics); Antipsychotics (neuroleptics, etc.); Antiandrogens (GnRH analogues and antagonists), and Recreational drugs) and/or psychogenic such as generalised type (e.g., lack of arousability and disorders of sexual intimacy) or Situational type (e.g., partner-related, performance-related issues or due to distress) [26].

Interesting observations were reported in case-control study prepared by Chung et al. [27]. In this study, authors estimated the association between erectile dysfunction and having previously been diagnosed with psoriasis. The study group consist of 4,606 patients with erectile dysfunction, and randomly selected 13,818 controls. In this study, $136(0.7 \%)$ patients with psoriasis were reported, but dominant percentage of this patients was observed in erectile dysfunction group ( $n=77 ; 1.7 \%$ of the cases) than in control group $(n=59 ; 0.4 \%$ of controls). This results highlight a need for doctors to be alert to the development of erectile dysfunction in psoriasis patients.

In other study, erectile dysfunction in psoriasis patients was shown too. Cabete et al. [28] prepared study with 135 psoriasis patients and 201 controls. To assess the erectile dysfunction, the 5 -item version of the International Index of Erectile Function was used. This authors showed that psoriasis patients $(61.5 \%)$ had a higher prevalence of erectile dysfunction than controls (43.8\%), and an increased risk of more severe forms of erectile dysfunction.

The similar observation was reported in Tasliyurt et al. [29] study among 37 male psoriasis patients and 28 controls. In this study, erectile dysfunction was evaluated by using International Index of Erectile Function (IIEF) Scale. Authors showed that erectile dysfunction, were more common in in psoriasis patient group $(\mathrm{p}=0.018)$ than controls.

\section{Sexual behaviour}

The analysis of sexual behaviour in psoriasis was prepared by Armstrong et al. in two publication. First carried out in men [30], and second in case of women [31]. These authors analysed data from the National Health and Nutrition Examination Survey (NHANES) from 2003 to 2006 and 2009 to 2010.

In the case of men, the study group consisted of 6,444 U.S. men, but only 170 (2.6\%) had diagnosis of psoriasis. In context of sexual orientation, there were $95.5 \%$ of heterosexual men, and $4.5 \%$ of nonheterosexual men. It should be noted that the multivariate analysis has shown that psoriasis was not associated with differences in sexual orientation (odds ratio 1.78, 95\% confidence interval $[\mathrm{CI}] 0.75-4.15)$. The results of this study provided valuable information about heterosexual men with psoriasis sex life. It was observed that about heterosexual men with psoriasis were characterised by first sexual encounter at an earlier age than those without psoriasis. Additionally, they have significantly fewer lifetime female oral sexual partners compared with controls. In the case of nonheterosexual men, no significant differences were reported in terms of age 
first had sex, number of sexual partners, or frequency of unprotected sex [30].

In the case of women, the study group consist of 3,462 women, but only 92 had diagnosis of psoriasis. In context of sexual orientation, the multivariate analysis has shown that psoriasis was not associated with differences in sexual orientation (odds ratio [OR] 0.90, 95\% confidence interval [CI] 0.62-2.01). The most important conclusion of this study was observation that psoriasis was associated with a significantly reduced number of sexual partners in nonheterosexual women (rate ratio [RR] $0.11,95 \%$ CI $0.04-0.33, \mathrm{P}=0.001$ ). In the context of heterosexual women, no differences was reported in terms of age of first sexual encounter, number of lifetime male sexual partners, and number of lifetime male oral sex partners. The authors emphasized that heterosexual women with psoriasis had 1.13 times more unprotected sex (RR 1.13, 95\% CI 1.02-1.24, $\mathrm{P}=0.03$ ) compared with those without psoriasis. Contrary to men, women sexual behavior based on sexual orientation could be more modified by psoriasis than in case of men [31].

It should be stressed that there is a lot of studies which analysed the sexual problem patients with psoriasis. However, only few publications have highlighted this problematic in patients with other dermatological diseases such as acne, atopic dermatitis, chronic hand eczema, and systemic sclerosis.

\section{Acne}

Acne is one of the most common chronic inflammatory disorder of the skin. This disease is most commonly in adolescence, but the prevalence of acne in the adult population is increasing. The researchers point that major aetiologic factors of acne are: I. hormonal imbalance, II. excess of sebum excretion, III. hyper-proliferation of follicular keratinocytes and bacteria [32].

Acne could be a serious problem in interpersonal relationships among teenagers. In the context of human development process, adolescence is a period of transition from childhood to adulthood. It is particularly important because of changes from dependence on adult direction and protection to self direction and self determination. Psychologists stress that adolescent development is very important step in creation of the interpersonal relationship with peers for socialization, friendship and support. In this case, adolescents must learn to cope with psychological stress, handle peer pressure, deal with their emotions, resolve conflicts, as well as build bridges with friends and family. The disease in adolescence may hinder the development of this process, especially if symptoms are visible.

It is still not known much about sexual dysfunctions in acne. Because of this, key research priorities in studies about sexual dysfunction in acne include development of robust, long-term, prospective population epidemiology studies to determine the prevalence of sexual dysfunction in acne; and examination of the role of environmental factors in the development and exacerbation of this dysfunctions.

\section{ATOPIC DERMATITIS}

Atopic dermatitis is a chronic inflammatory skin disorder which is characterized by eczema, pruritus and cutaneous hyperactivity to allergic triggers. Physical symptoms of this disorders may include dryness, redness, swelling, flaking, blistering, cracking, intense itching and bleeding. Atopic dermatitis can also lead to many psychological problems, for instance emotional stress, low self-esteem, mood changes, as well as depression. It should be noted that embarrassment as a result of their appearance can lead to social isolation. For this reason, it could also influence on sexual functions [33].

There is still no study about the relationship between sexual dysfunctions and atopic dermatitis (AD). Probably, atopic dermatitis can have negative influence on human psychosexual development, especially among adolescents. Epidemiological statistics show that this skin disease is very common in young person [33].

Actually, there is one study prepared by Shiu-Dong Chung et al. [34] in 3,997 patients with newly diagnosed ED. This study was carried by using data from the Taiwan National Health Insurance program. Authors observed that there was an association between $\mathrm{ED}$ and prior AD. However, the problematic of other sexual dysfunction in atopic dermatitis is out focus.

In the context of erectile dysfunction, there was very important study which was prepared by Kimata [35]. The aim of this paper was assessed the effects of viewing humorous films on erectile dysfunction in patients with atopic dermatitis. The effectiveness of this humorous films was evaluated in randomly assigned 18 patients 
and their healthy wives by using International Index Erectile Function (IIEF) domain (erectile function, orgasmic function, sexual desire, intercourse satisfaction, overall satisfaction). The result of this study showed that viewing humorous films significantly improved the IIEF domain, especially in context of increased serum testosterone levels and decreased serum estradiol levels. However, this effect was short-term. For this reason, there is need to prepare other empirical studies in this domain.

\section{CHRONIC HAND ECZEMA}

Hand eczema is dermatological diseases causing emotional and physical distress. This inflammatory skin disease is characterized by erythema, oedema and sometimes vesicles in the acute phase, but in the chronic state the skin changes are dominated by infiltration, scaling and fissures [36].

The problematic of sexual function in chronic hand eczema needs also much more empirical studies. Ergün et al. [37] analyzed sexual functions in Turkish hand eczema. In this case, the study group consist of 91 female (43 patients vs. 48 controls) and 79 male (45 patients vs. 34 controls). The Female Sexual Function Index (FSFI) and the International Index of Erectile Function (IIEF) were used to assess sexual function. The results of the study demonstrated that patients with hand eczema had sexual dysfunction. Moreover, International Index of Erectile Function total score was also found to be significantly decreased in male patients compared with controls.

\section{Research Gaps in Sexual Dysfunction in Skin Disorders: Opportunities for Future Studies}

First of all, there are not studies about sexual problem in skin disorders in context of adolescence. It is known that skin changes influence both on personality development, and psychosexual development.

Secondly, there are too small number of papers about relationship between some prescription drugs and patients' sexual function. This factor could have a significant impact on the therapy effectiveness.

Thirdly, this review shows that there is still too small publication about sexual dysfunction in skin disorders, especially other than psoriasis. Qualitative and in-depth quantitative research, including longitudinal studies, are needed on issues for which routine survey data leave many unanswered questions.
Fourthly, it should be stress that there are too small papers about patients' expectations about patient - doctor relationship in context of medical interview.

\section{REFERENCES}

1. Freedberg IM, Eisen AZ, Wolff K, Austen KF, Goldsmith LA, Katz SI. Fitzpatrick's Dermatology in general medicine. $6^{\text {th }}$ edition. New York: McGraw-Hill; 2003.

2. Barankin B, DeKoven J. Psychosocial effect of common skin diseases. Canadian Family Physician. 2002;48:712-6.

3. Garg A, Grant-Kels JM. Ethical considerations in dermatology residency. Clin Dermatol. 2012;30:202-9.

4. Owczarek K, Jaworski M. Quality of life and severity of skin changes in the dynamics of psoriasis. Adv Dermatol Allergol. 2016;33:102-8.

5. de Korte J, Sprangers MA, Mombers FM, Bos JD. Quality of life in patients with psoriasis: a systematic literature review. J Investig Dermatol Symp Proc. 2004;9:140-7.

6. Davis LS. Psycho dermatology: the psychological impact of skin disorders. JAMA. 2007;297:97-8.

7. Locala JA. Current concepts in psycho dermatology. Curr. Psychiatry Rep. 2009;11:211-8.

8. WHO-Department-of-Reproductive-Health-and-Research. Report of a technical consultation on sexual health. [WWW document] 2006. Geneva, Switzerland. URL http://www.who.int/ reproductivehealth/publications/sexual_health/defining_sexual_ health.pdf?ua=1 (last accessed: 01 June 2014).

9. Meeuwis KA, de Hullu JA, Nieuwenhof HP, Evers AW, Massuger LF, van de Kerkhof PC, et al. Quality of life and sexual health in patients with genital psoriasis. Br J Dermatol. 2011;164:1247-55.

10. Lowes MA, Suárez-Fariñas M, Krueger JG. Krueger. Immunology of Psoriasis. Ann Rev Immunol. 2014;32:227-55.

11. Palijan TZ, Kovacevic D, Koic E, Ruzic K, Dervinja F. The impact of psoriasis on the quality of life and psychological characteristics of persons suffering from psoriasis. Coll Antropol. 2011;35(Suppl. 2):81-5.

12. Rieder E, Tausk F. Psoriasis, a model of dermatologic psychosomatic disease: psychiatric implications and treatments. Int J Dermatol. 2012;51:12-26.

13. Kurizky PS, Mota LM. Sexual dysfunction in patients with psoriasis and psoriatic arthritis--a systematic review. Rev Bras Reumatol. 2012;52:943-8.

14. Wylie G, Evans CD, Gupta G. Reduced libido and erectile dysfunction: rarely reported side-effects of methotrexate. Clin Exp Dermatol. 2009;34:e234.

15. Aguirre MA, Vélez A, Romero M, Collantes E. Gynecomastia and sexual impotence associated with methotrexate treatment. J Rheumatol. 2002;29:1793-4.

16. Chen YJ, Chen CC, Lin MW, Chen TJ, Li CY, Hwang CY, et al. Increased risk of sexual dysfunction in male patients with psoriasis: a nationwide population-based follow-up study. J Sex Med. 2013;10:1212-8.

17. World Health Organization. ICD-10: International statistical classification of diseases and related health problems. Geneva: World Health Organization; 1992.

18. Molina-Leyva A, Almodovar-Real A, Ruiz-Carrascosa JC, Molina-Leyva I, Naranjo-Sintes R, Jimenez-Moleon JJ. Distribution pattern of psoriasis, anxiety and depression as possible causes of sexual dysfunction in patients with moderate to severe psoriasis. An Bras Dermatol. 2015;90 338-45.

19. Molina-Leyva A, Almodovar-Real A, Ruiz-Carrascosa JC, Naranjo-Sintes R, Serrano-Ortega S, Jimenez-Moleon JJ. Distribution pattern of psoriasis affects sexual function in moderate 


\section{www.odermatol.com}

to severe psoriasis: A prospective case series study. J Sex Med. 2014;11:2882-9.

20. Ji S, Zang Z, Ma H, Gu M, Han Y, Wang L, et al. Erectile dysfunction in patients with plaque psoriasis: the relation of depression and cardiovascular factors. Int J Impot Res. 2016;28:96-100

21. Maaty AS, Gomaa AH, Mohammed GF, Youssef IM, Eyada MM. Assessment of female sexual function in patients with psoriasis. J Sex Med. 2013;10:1545-8.

22. Sampogna F, Gisondi P, Tabolli S, Abeni D. Impairment of sexual life in patients with psoriasis. Dermatology. 2007;214:144-50.

23. Al-Mazeedi K, El-Shazly M, Al-Ajmi HS. Impact of psoriasis on quality of life in Kuwait. Int J Dermatol. 2006;45:418-24.

24. Ahmed A, Leon A, Butler DC, Reichenberg J. Quality-of-life effects of common dermatological diseases. Semin Cutan Med Surg. 2013;32:101-9.

25. Guenther L, Han C, Szapary P, Schenkel B, Poulin Y, Bourcier M, et al. Impact of ustekinumab on healthrelated quality of life and sexual difficulties associated with psoriasis: results from two phase III clinical trials. J Eur Acad Dermatol Venereol. 2011;25:851-7.

26. Gratzke C, Angulo J, Chitaley K, Dai YT, Kim NN, Paick JS, et al. Anatomy, physiology, and pathophysiology of erectile dysfunction. J Sex Med. 2010;7(1 Pt 2):445-75.

27. Chung SD, Keller JJ, Chu TW, Lin HC. Psoriasis and the risk of erectile dysfunction: a population-based case-control study. J Sex Med. 2012;9 130-5.

28. Cabete J, Torres T, Vilarinho T, Ferreira A, Selores M. Erectile dysfunction in psoriasis patients. Eur J Dermatol. 2014;24:482-6.

29. Tasliyurt T, Bilir Y, Sahin S, Seckin HY, Kaya SU, Sivgin H, et al. Erectile dysfunction in patients with psoriasis: potential impact of the metabolic syndrome. Eur Rev Med Pharmacol Sci. 2014;18:581-6.
30. Armstrong AW, Harskamp CT, Schupp CW. Psoriasis and Sexual Behavior in Men: examination of the National Health and Nutrition Examination Survey (NHANES) in the United States. I Sex Med. 2014;11:394-400

31. Armstrong AW, Follansbee MR, Harskamp CT, Schupp CW. Psoriasis and sexual behavior in U.S. women: an epidemiologic analysis using the National Health and Nutrition Examination Survey (NHANES). J Sex Med. 2013;10:326-32.

32. Steventon K, Cowdell F. Diet and acne: a review of the latest evidence. Dermatological Nursing. 2013;12:28-34.

33. Ahmed A, Leon A, Butler DC, Reichenberg J. Quality-of-life effects of common dermatological diseases. Semin Cutan Med Surg. 2013;32:101-9.

34. Chung S-D, Keller JJ, Lin H-C Association of erectile dysfunction with atopic dermatitis: A population-based case-control study. J Sex Med. 2012;9:679-85.

35. Kimata H. Short-term improvement of erectile dysfunction by viewing humorous films in patients with atopic dermatitis. J Sex Med. 2008;5:2107-10.

36. Thyssen JP, Johansen JD, Linneberg A, Menne T. The epidemiology of hand eczema in the general population-prevalence and main findings. Contact Dermatitis. 2010;62:75-87.

37. Egün M, Türel Ermertcan A, Öztürkcan S, Temeltaș G, Deveci A, Dinç G. Sexual dysfunction in patients with chronic hand eczema in the Turkish population. J Sex Med. 2007;4:1684-90.

Copyright by Mariusz Jaworski. This is an open-access article distributed under the terms of the Creative Commons Attribution License, which permits unrestricted use, distribution, and reproduction in any medium, provided the original author and source are credited.

Source of Support: Nil, Conflict of Interest: None declared. 